\title{
The processing of positional information in a two-item sequence limits the emergence of symmetry in baboons (Papio papio), but not in humans (Homo sapiens)
}

\author{
Joël Fagot ${ }^{1,2}$ - Raphaelle Malassis ${ }^{1,2}$ - Tiphaine Medam ${ }^{1,2}$
}

Published online: 4 August 2017

(C) Psychonomic Society, Inc. 2017

\begin{abstract}
When trained to associate Stimulus A to Stimulus $\mathrm{B}$, humans can derive the untrained symmetrical $\mathrm{B}$ to A relation while nonhuman animals have much more difficulties. Urcuioli (2008, Journal of the Experimental Analysis of Behavior, 90, 257-282; 2015, Conductal, 3, 4-25) proposed that the apparent difficulty of animals in symmetry testing reflects their double encoding of the information on the stimuli (identity and relation) and their positional (i.e., spatial and temporal/ordinal) characteristics. This comparative study tested the emergence of symmetry in humans and baboons in a task in which the position of the stimuli was manipulated independently of their relation. Humans and baboons initially learned to associate pairs of visual shapes on a touch screen in a specific order. Three pairs of (A-B, C-D, and E-F) stimuli were used in training. After training, the two species were tested with the B-A, F-C, and E-D pairs. The B-A pairs preserved the association initially learned with A-B but reversed the positional information relative to training. The F-C pair neither preserved the association nor the positional information of the training pairs, and positional information were the only cues preserved in the E-D pair. Humans showed a response time advantage for B-A, suggesting symmetry, but also for E-D, suggesting that they also process positional information. In baboons, the advantage was found only for E-D, suggesting that they only process positional information. These
\end{abstract}

Joël Fagot

joel.fagot@univ-amu.fr

1 Laboratory of Cognitive Psychology, Aix Marseille University, CNRS, Marseille, France

2 Brain and Language Research Institute, Aix Marseille University, CNRS, Aix-en-Provence, France results confirm that the processing of stimulus pairs differ between nonhuman animals to humans.

Keywords Associative learning · Equivalence classes . Language $\cdot$ Categorization

Derived relational responding is a cognitive phenomenon in which trained relations between arbitrary stimuli result in the spontaneous emergence of untrained relations. This cognitive phenomenon is best demonstrated in humans by experiments on stimulus equivalence (Sidman \& Tailby, 1982). In such experiments, the participants must typically learn by trial and error that the visual sample Stimulus A must be associated in a conditional matching-to-sample task to the comparison Stimulus B. They must also learn that the same B stimulus now used as sample in the matching task must be associated to the comparison Stimulus C. After such (A-B, B-C) training, the subjects demonstrate stimulus equivalence when they spontaneously exhibit the relation of reflexivity, symmetry, and transitivity. Reflexivity implies the ability to spontaneously associate each stimulus to itself, for instance, to indicate that A goes with A. The relation of symmetry implies that any given association learned during the training phase can be reversed. In other words, if the subject has learned that A goes to $\mathrm{B}$, he or she should be able to indicate without training that $\mathrm{B}$ also goes to $\mathrm{A}$. The relation of transitivity involves that the stimuli that have common associates go together. In other words, after being trained with A-B and B-C, the subject showing transitivity should be able to match $\mathrm{A}$ to $\mathrm{C}$ and $\mathrm{C}$ to A. Stimulus equivalence, as defined by the ability to process all these three relations, was initially theorized and demonstrated by Sidman and collaborators using this kind of matching-to-sample protocols (Sidman, 1971; Sidman \& Tailby, 1982), and the fact that our species has this ability 
has been largely confirmed since their initial studies (e.g., Adams, Fields, \& Verhave, 1993; Carp \& Petursdottir, 2015; Devany, Hayes, \& Nelson, 1986). One reason for which stimulus equivalence is interesting is that it appears to be closely linked with human language. First, our flexible use of words implies a bidirectional (i.e., symmetrical) relation between the word and its referent: the word refers to the object (e.g., in requesting contexts) as the object evokes the word (e.g., in naming contexts). Second, stimulus equivalence emerges at approximately 2 years of age (e.g., Boelens, van den Broek, \& van Klarenbosch, 2000; Lipkens, Hayes, \& Hayes, 1993) and is thus concurrent with language learning. Third, children with language disabilities or other clinical groups with linguistic deficiencies (e.g., children with autism) often express difficulties in tasks of stimulus equivalence (Devany et al., 1986; Eikeseth \& Smith, 1992; O'Donnell \& Saunders, 2003; but see Carr, Wilkinson, Blackman, \& Mcllvane, 2000). The apparent relations between language and stimulus equivalence call for comparative studies on stimulus equivalence in nonlinguistic species.

From a comparative point of view, stimulus equivalence has been an elusive phenomenon when tested in nonhuman animals, such as pigeons (e.g., R. Lipkens, Kop, \& Matthijs, 1988) and nonhuman primates (e.g., rhesus monkeys and baboons; Sidman, Rauzin, et al., 1982). Positive results have been repeatedly reported regarding reflexivity (Barros, Galvão, \& McIlvane, 2002; Sweeney \& Urcuioli, 2010) and transitivity (D'Amato, Salmon, Loukas, \& Tomie, 1985; Kuno, Kitadate, \& Iwamoto, 1994; Zentall, Clement, \& Weaver, 2003), but the large majority of the studies have reported negative results regarding the symmetry relation. Lionello-DeNolf (2009) reviewed 24 investigations of symmetry in nonhuman animals and concluded that only three of these studies found clear evidence of symmetry in their subjects (pigeons: Frank \& Wasserman, 2005; sea lions: Kastak, Schusterman, \& Kastak, 2001; Schusterman \& Kastak, 1993). Since this review has been published, a few additional papers (e.g., Urcuioli, 2015) have confirmed that symmetry is in the scope of pigeons. Nevertheless, the general picture of the existing literature remains that symmetry seems an exceptionally difficult feat in nonhuman species. The main goal of our research was to identify which factor may best explain the general difficulties expressed by nonhuman animals during symmetry testing.

Urcuioli $(2008,2015)$ proposed that the difficulty experienced by pigeons in symmetry testing would result from their mode of encoding of two types of information that are (1) the information on the identity of the nominal stimuli and their relation and (2) the information on their position in the spatial and temporal dimensions. According to Urcuioli, a Stimulus A appearing as sample in a fixed spatial position \#1 (e.g., typically, the center response key) would be encoded as Stimulus A in temporal/ordinal position \#1 at spatial position
\#1, while a Stimulus B appearing as a comparison in a fixed spatial position \#2 (e.g., left or right response key) would be encoded as Stimulus B in temporal/ordinal position \#2 at spatial location \#2. This mode of encoding would prevent generalization in symmetry trials because Stimulus A in temporal/ ordinal position \#1 and spatial position \#1 would be treated as functionally different from the same stimulus presented in the temporal/ordinal position \#2 and spatial position \#2.

The idea that nonhuman animals focus too strongly their attention on positional information during the learning of associations between pairs of items is mostly supported by Frank and Wasserman's (2005) and Urcuioli's (2008, Experiment 3) studies in pigeons. These studies had two interesting distinguishing features that may explain their positive results in symmetry trials. First, they both used a successive matching-to-sample task rather than the two-alternative matching tasks (e.g., Lipkens et al., 1988). In their successive matching task, the sample and comparison stimuli are shown on the same location on the screen, and the pigeons have to peck (go response) or not (no-go response) the comparison stimulus depending on the identity of the sample. This aspect of their task contrasts strongly with the two-alternative matching-to-sample task in which the sample and comparisons are shown on different locations on the displays (see Lionello-DeNolf, 2009, for a review of negative findings obtained in this task; see also Urcuioli, 2008, Experiments 1 and 2). Second, both Frank and Wasserman (2005) and Urcuioli (2008, Experiment 3) mixed identity (A-A, B-B) and symbolic matching trials (A-B) during the training phase. One consequence of this intermixing of the identity and symbolic matching trials is that each stimulus has also been presented in the first or second temporal/ordinal position during training. This counterbalancing might have reduced the importance given to temporal/ordinal information in the pigeons' mode of encoding, allowing a stronger focus on relational information at the origin of a successful performance in symmetry trials. Said differently, the two most convincing lines of evidence of symmetry in nonhuman animals were obtained with a test design that minimized the possibility to encode positional (spatial and temporal/ordinal) information in pigeons. This hypothesis may also explain why symmetry training, involving exposure to the reversal of the initially learned associations, seems to promote generalized symmetry (e.g., Pepperberg \& Gordon, 2005; Schusterman \& Kastak, 1993).

Our study aimed at testing further the general idea that nonhuman animals encode in priority the positional information to the detriment of the relational information in A-B types of sequences, and that this mode of encoding is the main source of failures in symmetry tests involving B-A types of trials. We developed for that purpose a novel prediction task, inspired from the serial response time task (Misyak, Christiansen, \& Bruce Tomblin, 2010; Nissen \& Bullemer, 1987), which was given to both humans (Homo sapiens) and 
Guinea baboons (Papio papio). The main advantage of this procedure is to offer fine-grained measures of participants' expectations on critical trials rather than merely reflecting the result of a decision process in a binary choice task, as in the aforementioned studies. Our task required participants to learn in a training phase the association between items in three pairs of shapes. A test phase then presented some novel pairings of the stimuli used during the training phase. One test condition preserved positional information but disrupted relational information. One other test condition preserved relational information but disrupted positional information. A last test condition disrupted both kinds of information. We predicted that the baboons will demonstrate an advantage for the stimulus pairs preserving positional information, while humans would demonstrate an advantage for the stimulus pair preserving the relations. To our knowledge, this study is the first to extend Urcuioli's line of research with nonhuman primate species.

\section{Method}

\section{Participants}

Data were collected on a total of 14 Guinea baboons (Papio papio; age range: 4-12 years; seven females) living in a larger social group of 24 individuals located at the CNRS Station de Primatologie, Rousset-sur-Arc, France. This group of baboons is maintained within a $700 \mathrm{~m}^{2}$ outdoor enclosure connected to an indoor area used at night, and two test rooms in which the test systems were presented ad libitum (see below and Fagot \& Bonté, 2010, for a more detailed description of their living conditions). The baboons were all marked with a radiofrequency identification (RFID) microchip implanted in each arm. Water was provided ad libitum during the experiment. Animals were not food deprived. They received their regular portion of food (monkey chow and fruits) every day at 5 p.m. All baboons had previously participated to numerous computerized experiments using the ALDM test systems and had already been involved in an unpublished experiment that used the same prediction task as in this study. However, this previous experiment never trained or tested our baboons with symmetrical pairs. Note also that some of our subjects have already been involved in Medam, Marzouki, Montant, and Fagot's (2016) study on the effect of category training on symmetry. The use of a two-alternative matching-to-sample procedure in this study failed to reveal symmetry in these individuals.

The human data were collected on a total of 21 adults (age range: 19-28 years, 16 females) who were recruited at our university and participated for payment. These participants were all unaware of the purpose of the research and have never been involved in any of our research programs.

\section{Apparatus}

The baboons had an unrestricted access during the experiment to 10 automated learning devices for monkeys (ALDMs; Fagot \& Bonté, 2010; Fagot \& Paleressompoulle, 2009), which were installed in two trailers directly accessible from their outside enclosure. Each ALDMs consisted of a $70 \mathrm{~cm} \times$ $70 \mathrm{~cm} \times 80 \mathrm{~cm}$ testing chamber with a view port and two hand ports. The view port allowed to see a 19-in. touch screen (1939L Open-Frame Touchmonitor, Elo Touch Solutions) installed in front of the chamber, and the hand port was used to manipulate it. The RFID microchip implanted in the arm of each subject served to automatically identify each baboon once it introduced one of its arms through the hand port. The baboon could use the ALDM test system of its choice, whenever it wanted to use it, because the automatic reading of the subject identity was used to prompt the program to resume the trial list at the appropriate place for that subject. The ALDMs automatically dispensed several grains of dry wheat inside the test chamber once a correct response had been registered. Incorrect responses were not food rewarded but gave rise to a 3-s time-out indicated by a green screen. The experiment was programmed using E-Prime (Version 2.0, Psychology Software Tools, Pittsburgh, PA) software.

The apparatus for humans consisted of a 19-in. computer touch screen (ET1915L, Elo Touch) installed on a table in a quiet room. Correct responses in the task were indicated by a short 1-s black screen; incorrect responses were followed by a 3 -s time-out with a green screen.

\section{Stimuli}

This experiment used a total of six different yellow visual shapes that served to create three different pairs of stimuli (hereafter referred to as pair A-B, C-D, and E-F). The individual stimuli were shown on a $1024 \times 768$ pixel background and measured approximately $180 \times 180$ pixels on the screen. The stimuli were drawn for the purpose of the experiment, with the sole constraint that the discrimination of the items of the set should be easy for the two species.

\section{General design of the task}

The two species were tested in an adapted version of the serial reaction time task, in which the subjects had to select and touch two stimuli consecutively. The procedure is illustrated in Fig. 1. Each trial began with a display of two items presented one above the other (locations balanced) on the left side of the screen. These two stimuli were first presented during 200 $\mathrm{ms}$, after which one of them disappeared for a short period of $80 \mathrm{~ms}$. This short disappearance of the stimulus gives the impression that the stimulus blinks once. The stimulus disappearing for $80 \mathrm{~ms}$ was the positive stimulus $(\mathrm{S}+)$ that 


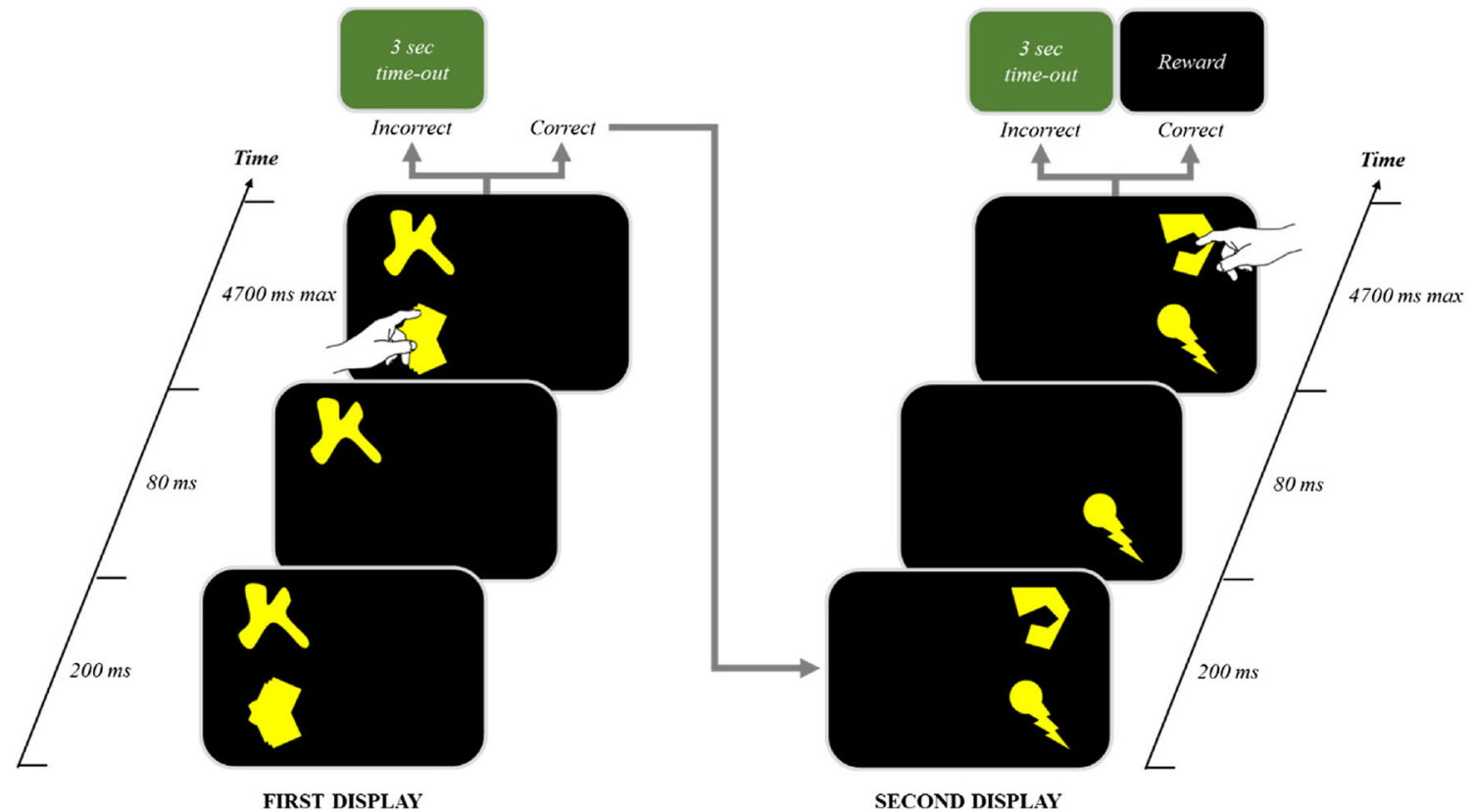

Fig. 1 Illustration of the experimental procedure used in baboons. This same procedure was also used in humans, except that humans did not receive a food reward after each successful response

the subject had to touch in this first display. A total of $4,700 \mathrm{~ms}$ was allowed for touching this stimulus. A correct touch of S+ at this stage caused the display to disappear immediately to be replaced by the second display. An incorrect selection of the negative stimulus ( $\mathrm{S}-$ ) triggered a 3-s green screen, and the trial was aborted and scored as incorrect. Once the S+ had been touched, the subject saw the second display containing two other stimuli that were presented one above the other (location balanced) on the right part of the screen. Again, one of these two stimuli disappeared for $80 \mathrm{~ms}$, and the subject then had to touch this S+ stimulus, while avoiding S-. Again, 4,700 ms were allowed for responding at this stage. A correct selection of S+ on this second display triggered a positive feedback (a black screen and food reward for monkey, and black screen only for humans), and an incorrect response produced the 3-s green time-out screen, indicating that an error has been made. Trials in which the participants failed to touch the screen during the time window allotted for responding to the first or second display were systematically aborted. These aborted trials were represented immediately.

The procedure outlined above was used in a first training phase and in the following test phase. The pairs used in each phase are indicated in Table 1. The training phase aimed to teach the subjects that the stimuli were organized in pairs. In the training trials, each first stimulus of a given pair (e.g., Stimulus A in the A-B pair) served as $\mathrm{S}+$ in the first display of the training trials using this pair, and the second stimulus of the pair (i.e., B) served as $\mathrm{S}+$ in the second display. The first and second stimuli of another pair (e.g., C-D or E-F pair) served as $\mathrm{S}-$ in the first and the second displays, respectively.
The test sessions presented the same stimuli as in training sessions but used the three stimulus pairings indicated in Table 1. The first one (i.e., B-A) tested for the emergence of symmetry, because this pairing is a reversal of the A-B pairing of the training phase. The remaining two parings (i.e., F-C and E-D) assessed the effect of stimulus ordering, because the position of these stimuli was either preserved (E-D) or reversed (F-C) in comparison to training. Within a trial, the first and second stimulus of a given pair (e.g., Stimuli B and A, in the B-A pair) served as $\mathrm{S}+$ in the first and second display, respectively, while the first and second stimulus of another pair (e.g., E-D or F-C pair) served as S- in the first and the second displays, respectively. The dependent variables used for statistical analyses were the number of correct responses to the first and the second display of each kind of trial, as well as the response times to $\mathrm{S}+$ in the first (hereafter referred to as

Table 1 Experimental design in the training and test phase

\begin{tabular}{llll}
\hline Training Pairs & Test Pairs & Associative cue & Positional cue \\
\hline A-B & B-A & + & - \\
C-D & E-D & - & + \\
E-F & & & - \\
C-D & F-C & - & \\
E-F & & & \\
\hline
\end{tabular}

Note. Each letter corresponds to one stimulus in the set. For each pair, the first letter indicates the stimuli used for the first display, and the second letter indicate the stimulus used for second display. Bold characters indicate the stimulus of the training pairs under scrutiny in the corresponding test pair. A "+" sign indicates that the associative (third column) and/or positional cues (fourth column) present in the training pair were preserved in the test pairs. A "-" sign indicates that it was not. 
RT1) and second display of the trials (RT2). However, because very few errors were recorded in the task, our attention was mostly focused on response times variations that provided fine-grained information on learning processes and on the effect of the different tested factors. With this test design, comparison of the response to the second display of the B-A and $\mathrm{F}-\mathrm{C}$ test pairs should reveal if the participants process the trained associations as if they are symmetrical. If they do so, then they should express a better accuracy on the second object and/or faster RT2 for the A shape than for the C shape. In addition, a comparison of the response to the second display of the F-C and E-D pairs should also indicate if the subject process the stimulus position, independently of the trained associations. In that case, they should express a better accuracy and/or faster RT2 in D than in C.

\section{Training procedure}

During training, the human and baboon participants received blocks of 96 counterbalanced training trials, each containing 32 trials with the A-B, C-D, and E-F stimulus pairs. Each pair was presented in an identical number of trials as the $\mathrm{S}+$ or the $\mathrm{S}-$ pair within a block. The ordering of the trials was randomized at the beginning of each block and therefore differed from one block to the next. The baboons received a total of $20 \mathrm{such}$ blocks (1,920 trials) during the training phase. To avoid boredom or inattention to the task, the human participants only received five training blocks (480 trials).

Before the experiment, the baboons received two preliminary training phases, which both used different stimuli from those of the next training and testing phase. These two preliminary training phases were not presented to humans, who rapidly discovered by themselves that they had to touch the stimuli that blinked in the displays. Thus, in a first preliminary phase, the baboons were taught to touch the stimulus among two which disappeared for $80 \mathrm{~ms}$ from the screen. Once they reached $80 \%$ correct with two individual shapes, we proceeded to the second preliminary training phase. This phase introduced two displays in succession, which were presented following the same procedure as for the training trials (see above), but which used different stimuli from those of the final training and test phase. To obtain a reward, the baboon had to touch the stimulus that blinked once in each display, once again until they reached $80 \%$ correct with this design. The stimuli were organized in three pairs in this second preliminary training phases, to teach the subjects that the task involved some associations between stimuli. The training phase involving the three A-B, C-D, and E-F pairs was introduced immediately after these two preliminary training phases. The instruction given to humans prior to the experiments only explained that they had to touch some stimuli on the screen, and that response errors are indicated by a green screen.

\section{Test procedure}

During the test phase, the baboons received 10 test blocks of 96 trials each (960 trials). Each block involved 32 trials with each of the three (B-A, F-C, and E-D) test pairs. Human participants received five test blocks (480 trials). Importantly, the stimulus taking the role of the A, B, C, D, E, and F stimulus was counterbalanced across subjects. This counterbalancing of the visual shapes guaranteed that the variations in accuracy or response time are not tied to individual stimuli.

At the end of the test phase, the experimenter asked the human subjects to report verbally their understanding of the task. Thus, all the subjects were first asked, "What were you supposed to do?" Then, if the free report of the subjects did not include the following information, the subject was asked to answer the following questions in the following order: (1) "Which forms had to be touched?" (2) "Did you notice some relationships between and among the stimuli?" (3) "Did you notice any change during the experiment?" and (4) "Did you use verbal names to refer mentally to the stimuli?"

\section{Data filtering}

A filtering procedure was applied for the analysis of correct response times to neglect the outlier trials with excessively long response times that may reflect transient inattention to the task. Thus, we first discarded from the data set all the trials in which RT1 and/or RT2 was greater than $3 \mathrm{~s}$. This first layer of filtering removed $.11 \%$ of the trials for humans and $.30 \%$ for the baboons. The second filter additionally removed all the trials for which RT2 was greater than two standard deviations from the RT2 mean (computed for each individual and block). This second filtering procedure removed $3.62 \%$ of the remaining trials for humans and $4.32 \%$ for the baboons. Statistical analyses considered separately the data from the two species because of an uneven number of training and test blocks in humans and baboons.

\section{Results}

\section{Humans}

The human participants performed consistently high in the task, with an average percentage of correct responses of 96.97\% $(S D=17 \%)$ in the training phase, and $96.4 \%(S D=$ $18.64 \%$ ) in the test phase. This performance precludes detailed statistical analyses on accuracy due to potential ceiling effects.

A preliminary inspection of the data indicated that some participants showed an acceleration in RT2 throughout the training phase, while others did not. These different response profiles suggest that only a subset of humans-those for 
which the RT2 declined with training - could predict their response to the second item of the pair depending on the identity of the first item. We therefore analyzed the postexperimental verbal reports of the subjects, and split our sample of participants in two groups. The first group ( $n=10$, hereafter named AS+), includes all the participants who have reported verbally during the debriefing that the stimuli were organized in pairs. The second group ( $n=11$, hereafter named the group $\mathrm{AS}^{-}$) includes all the subjects who failed to report any association between or among the stimuli.

Training phase Figure 2 represents the RT1 and RT2 training data for the two groups of participants.

A two-way analysis of variance (ANOVA) was computed for each group, using the stimulus pair (A-B, C-D, and E-F) as well as the training block (1-5) as independent variables, and RT1 as a dependent variable. None of the main effects or the interactions emerged as significant, in either group (all $p \mathrm{~s}>$ $.05)$. We found in particular no reliable effect of stimulus pair for RT1, whatever the group considered: AS $+F(2,18)=.17$, $p>.10, \eta_{\mathrm{p}}^{2}=.02 ; \mathrm{AS}-, F(2,20)=1.71, p>.10, \eta_{\mathrm{p}}{ }^{2}=.15$. This finding is important because it demonstrates that the response to the first item of each pair did not differ in terms of perceptual or motor constraints.

The same ANOVAs conducted on the RT2 revealed no reliable main effects or interaction when the AS- group was considered (all $p \mathrm{~s}>.10$ ). In the case of the AS+ group, neither the effect of stimulus pair nor the interaction were significant, $F(2,18)=2.16, p>.1, \eta_{\mathrm{p}}{ }^{2}=.19 ;$ and $F(8,72)=1.07, p>.1$, $\eta_{\mathrm{p}}{ }^{2}=.11$, respectively. However, the main effect of block was statistically significant for this AS+ group, $F(4,36)=12.59, p$ $<.001, \eta_{\mathrm{p}}{ }^{2}=.58$. As shown in the top right panel of Fig. 2 , this main effect of block corresponds to a reduction of response

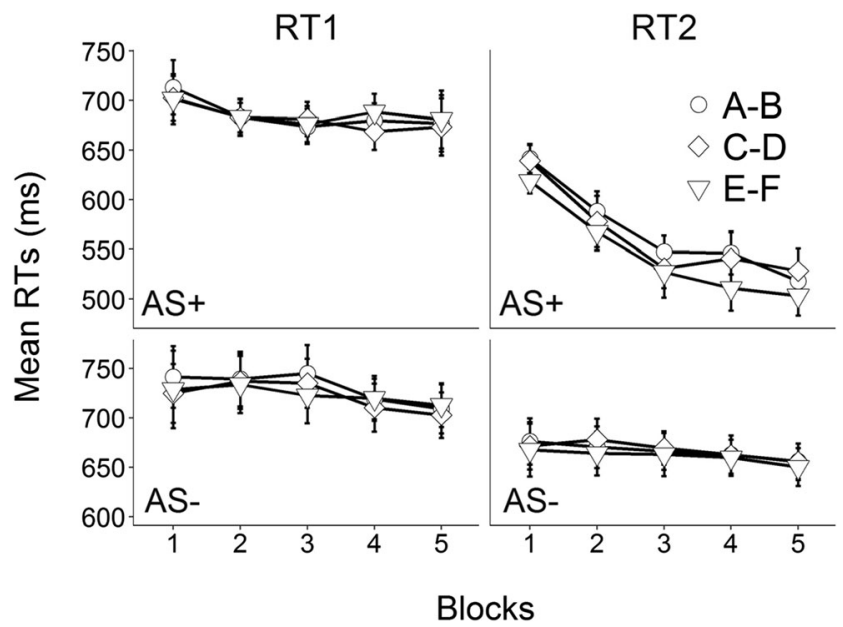

Fig. 2 Mean correct response times on the first (RT1) and the second (RT2) display obtained for each training pair, in the five training blocks presented to the human participants of the AS+ (top) and AS- (bottom) group. Note that the $y$-axes have different scales in the two groups and display only a subset of the range. Error bars indicate the standard errors centered on each participant mean RT times with repeated training. One potential explanation of this decrease in RT2 is that it reflects a general improvement of performance for pointing the stimuli on the screen, but this hypothesis is ruled out because our analyses of RT1 showed that they remained stable across the training blocks. These findings, along with the verbal reports of the subjects, therefore suggest that $\mathrm{AS}+$ is the sole group that learned that the stimuli were organized by pairs. For these individuals, the learning of the stimulus pairing that occurred across the training blocks gave rise to reduced RT 2 because the identity of the second stimulus became progressively predictable with a continuous exposure to the training stimulus pairs.

Test phase Figure 3 represents the RT2 data obtained during the test phase in the AS+ and $\mathrm{AS}-$ groups.

Analyses for the AS+ group With our test procedure, we expected that the effects of symmetry (if any) will emerge in the AS+ group immediately after the different pairs were recombined, therefore during the first test trials. Two-tailed paired $t$ tests compared the mean RT2 for the first 10 trials of each pair. These analyses revealed that the response times were shorter on average for the B-A pair (mean $=584 \mathrm{~ms}, S D$ $=57 \mathrm{~ms})$ than for the F-C pair $(620 \mathrm{~ms}, S D=60 \mathrm{~ms}), t(9)=$ $3.22, p<.05$, Cohen's $d=.81$. Importantly, the stimuli composing the B-A and F-C test pairs all changed their position in comparison to training, and in these two pairs, only B-A preserved the association learned during the training phase. The shorter RT2 obtained for B-A, in comparison to F-C, can therefore be explained by the fact that the subject expected to see an A in the second display of the symmetrical B-A pairs, while no similar prediction was possible in the $\mathrm{F}-\mathrm{C}$ pairs. This

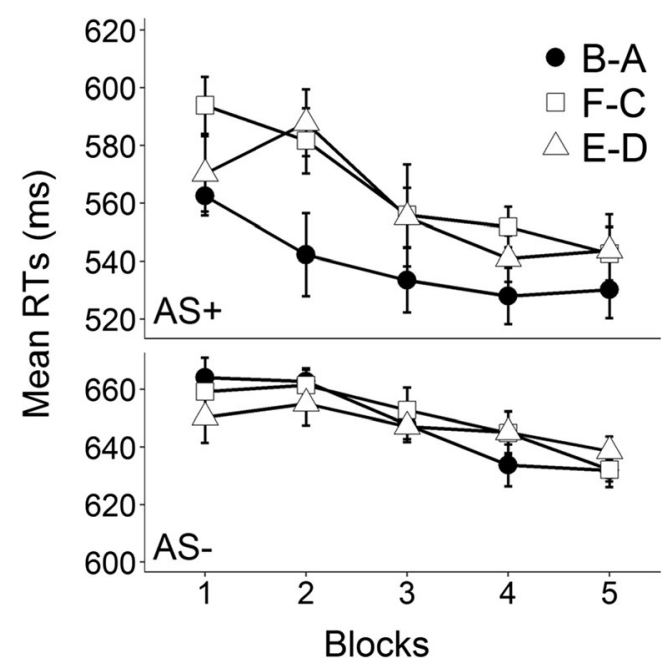

Fig. 3 Mean correct response times on the second display (RT2) obtained in the AS+ and AS- group for each test block and stimulus pair. Note that the $y$-axes have different scales in the two groups and display only a subset of the range. Error bars indicate the standard errors centered on each participant mean RT 
result suggests that the human participants from the AS+ group processed the relation of symmetry.

Data analyses also revealed shorter RT2 on average for the E-D pair (mean $=543 \mathrm{~ms}, S D=32 \mathrm{~ms}$ ), compared to both the $\mathrm{B}-\mathrm{A}, t(9)=-2.55, p<.05$, Cohen's $d=.47$, and the F-C pairs, $t(9)=-4.66, p<.01$, Cohen's $d=.49$. This effect indicates that the subjects from the AS+ group also processed the position of the stimuli, in addition to their processing of symmetry reported above.

Another question is if these effects could persist in AS+ with repeated exposure to the five test blocks. This question was addressed with a stimulus pair (B-A, E-D, F-C) by test block (1-5) two-way ANOVA on RT2. This analysis revealed that the main effect of test block was significant, $F(4,36)=$ $4.59, p<.01, \eta_{\mathrm{p}}{ }^{2}=.33$. It showed that the RT2 decreased on average with repeated testing. The effect of stimulus pair approached significance, $F(2,18)=3.53, p<.06, \eta_{\mathrm{p}}{ }^{2}=.28$, and the stimulus pair by test block interaction was not significant, $F(8,72)=1.12, p>.10, \eta_{\mathrm{p}}{ }^{2}=.11$. The effect of stimulus pair, albeit not significant, is shown in Fig. 3 by systemically shorter RT2 in the five test blocks for B-A than for both F-C and E-D. The fact the these RT2 remained systematically shorter during the five test blocks suggests a relative persistence of the effect of symmetry in our group of participants. Conversely, the initial advantage in RT2 found for the E-D pair during the analysis of the 10 first test trials disappeared rapidly. Figure 3 indeed indicates that RT2 for the E-D pair is shorter than the RT2 for the F-C pair in the first test block only. The effect of position seems therefore more transitory in humans of the AS+ group than the effect of stimuli symmetry.

Analyses for the AS- group The same set of analyses conducted on the AS- group revealed shorter response times in the 10 first trials on average for the E-D pair (mean $=642 \mathrm{~ms}$, $S D=96 \mathrm{~ms}$ ) than for the F-C pair (mean $=679 \mathrm{~ms}, S D=76$ $\mathrm{ms}), t(10)=2.36, p=.04$. This difference suggests an encoding of the position within the first 10 trials which is confirmed by a nonsignificant trend for shorter RT2 on average for E-D than for B-A (mean $=673 \mathrm{~ms}, S D=76 \mathrm{~ms}$ ), $t(10)$ $=2.05, p=.068$. No reliable difference emerged between B-A and F-C, $t(10)=0.39, p>.10$.

The ANOVA performed on the five blocks of the test phase revealed a main effect of block, $F(4,40)=5.07, p<.01, \eta_{\mathrm{p}}{ }^{2}=$ .37 , but no reliable effect of condition, $F(2,20)=0.14, p>$ $.100, \eta_{\mathrm{p}}{ }^{2}=.01$, and no significant interaction between these two factors, $F(8,80)=1.10, p>.100, \eta_{\mathrm{p}}^{2}=.10$. Taken together, results of the AS- group confirm that participants from the AS- group did not learn that the item that blinks once in the first display predicts a specific item in the second display. It seems that these participants encoded only the positional properties of the individual stimuli. However, as for the AS+ group, this encoding seems transitory as it only appeared in the first 10 test trials, but disappeared when the entire test phase was considered.

Verbal reports All the subjects from the AS+ group, but none of the AS- group, reported verbally after the experiment that they had noticed a change during the experiment (i.e., the changes between the training and testing phases). Within AS+, three subjects indicated that one association (i.e., for A-B) was preserved after the change. These data suggest variations among the AS+ subjects, some of them seem to process the symmetry relation implicitly, while the three subjects who noticed that the A-B association was preserved, processed it more explicitly. Despite these variations, all the AS+ subjects reported that they have given labels to the object stimuli.

\section{Baboons}

The baboons performed $76.30 \%$ correct on average during the training phase $(S D=42.48 \%)$ and therefore made more errors than humans. On that basis, we report below statistical analyses on both response times and accuracy.

Response times of the training phase We found earlier that the human subjects who noticed that the stimuli were organized in pairs during the training phase (AS+ participants) showed a progressive decline in RT2 during that phase. Because this decline in RT2 is in humans an indicator of learning, our first analyses of the baboons' data distinguished two groups of baboons. The first group (referred to below as the AS+ group) was composed of the individuals who showed reduced RT2 with training, and the second group (referred to below as the AS- group) was composed of the individuals who did not show this effect. This was achieved by way of one-tailed $t$ tests $(p<.05)$ comparing the mean RT2 obtained, for each individual, during the first and last blocks of the training phase. From these analyses, eight baboons were assigned to the AS+ group, the remaining six subjects were assigned to the AS- group.

Figure 4 represents the mean RT1 and RT2 obtained in the AS+ and AS- groups during training. Because the number of errors was greater in baboons than in humans, reducing the number of RT measures per block, statistical analyses of correct RT1 and RT2 considered blocks of 192 trials made from the grouping of two initial contiguous 96-trial blocks. RT1 and RT2 data were analyzed independently for the AS- and AS+ groups, using two-way ANOVAS, considering the stimulus pair (A-B, C-D, E-F) and the training block (ten 196-trial blocks) as independent variables.

For the AS+ group, the two-way ANOVA on RT1 revealed a main effect of block, $F(9,63)=3.40, p<.002, \eta_{\mathrm{p}}{ }^{2}=.33$. The main effect of stimulus pair and the interaction between the pair and block factors were not significant (all $p \mathrm{~s}>.10$ ). 


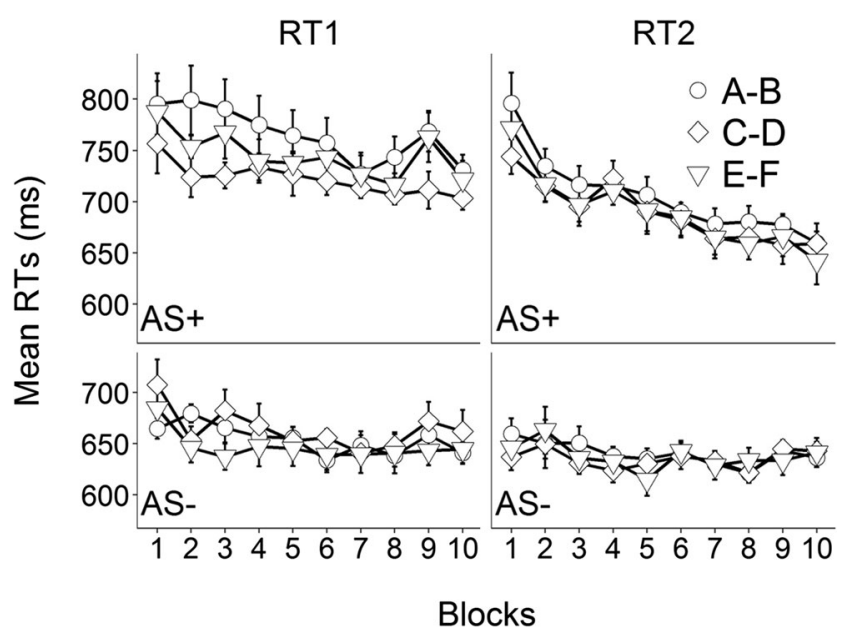

Fig. 4 Mean RT1 and RT2 obtained during the training phase for the AS+ and AS - groups of baboons. The $y$-axes have different scales in the two groups and display only a subset of the range. Error bars represent the standard errors centered on each participant mean RT

Similar results were obtained in AS+ for RT2, with a reliable effect of block being the only significant effect, $F(9,63)=$ $12.37, p<.001, \eta_{\mathrm{p}}^{2}=.64$.

For the AS- group, the two-way ANOVA conducted on RT1 showed a main effect of block, $F(9,45)=2.69, p<.02$, $\eta_{\mathrm{p}}{ }^{2}=.35$, but no reliable effect of stimulus pair and no stimulus pair by block interaction (all $p s>.1$ ). When computed on RT2, the same ANOVA revealed no reliable main effect or interaction in AS- (all $p \mathrm{~s}>.10$ ).

In sum, the individuals from the AS+ groups showed an effect of block for both RT1 and RT2. Regarding RT1, we can safely assume that the effect of block indicates a general effect of practice, reducing the response times with continuous training. Regarding RT2, the question arises of whether block effect only corresponds to a general effect of practice, as per RT1, or can also reflect the learning of the associations between the stimuli belonging to each pair. To address this question, we ran a complementary two-way ANOVA on the response times in which the training block (1-10) and the target of the response (Object 1 vs. Object 2) were used as factors. This ANOVA revealed a general effect of block, $F(9,63)=$ $10.36, p<.001, \eta_{\mathrm{p}}{ }^{2}=.60$, as well as a main effect of target, $F(1,7)=13.44, p<.01, \eta_{\mathrm{p}}{ }^{2}=.66$. Of the most importance for our purpose, these two factors interacted significantly, $F(9$, $63)=2.54, p<.05, \eta_{\mathrm{p}}{ }^{2}=.27$. As shown in Fig. 4 , training has a stronger effect on the time to respond to the second than to the first object of the stimulus pairs. This result is a strong indicator that the subjects of the AS+ group have learned that the stimuli are organized in pairs. By contrast, no decrease in RT2 was observed in AS-. This effect confirms that the AS+ and AS- groups behaved differently in the task, regarding their response to the second display. We will see below that these differences in behavior correspond to cognitive differences during the test phase.
Accuracy of the training phase Figure 5 reports the mean accuracy, for each group and training conditions. Following the same logic as above, the accuracy to the first object (Object 1) and second (Object 2) objects of the stimulus pairs were analyzed separately with block $(1-10)$ by stimulus pairs (A-B, C-D , E-F) two-way ANOVAs computed independently for the two groups of baboons.

For the AS+ groups, the two-way ANOVA on Object 1 only found a reliable effect of block, $F(9,63)=4.41, p<$ $.01, \eta_{\mathrm{p}}^{2}=.39$. A similar result was obtained when the response to Object 2 was considered, with the effect of block being the only significant effect, $F(9,63)=5.63, p$ $<.001, \eta_{\mathrm{p}}{ }^{2}=.43$. Because the effect of block was significant in AS+ for both Objects 1 and 2, the question arises of whether the increased accuracy with blocks for Object 2 can be accounted for only by a general effect of practice, as observed for Object 1, or if this effect can also be explained by the learning of the associations between the first and second stimulus of each pair. To assess this idea, we ran an additional block (1-10) by object (Object 1 vs. Object 2) two-way ANOVA, using accuracy as the dependent variable. This analysis indicated no main effect of object $(p>.10)$ but a main effect of block, $F(9,63)=6.65, p<.001, \eta_{\mathrm{p}}{ }^{2}=.49$, showing that the performance increased with training. More importantly for our purpose, the Object $\times$ Block interaction was also significant, $F(9,63)=2.68, p<.02, \eta_{\mathrm{p}}^{2}=.28$, indicating that the effect of block was stronger for Object 2 than for Object 1 . These findings therefore converge perfectly with those reported above on both RT1 and RT2. They confirm that the baboons from AS+ progressively learned the associations between the first and second objects of each stimulus pair.

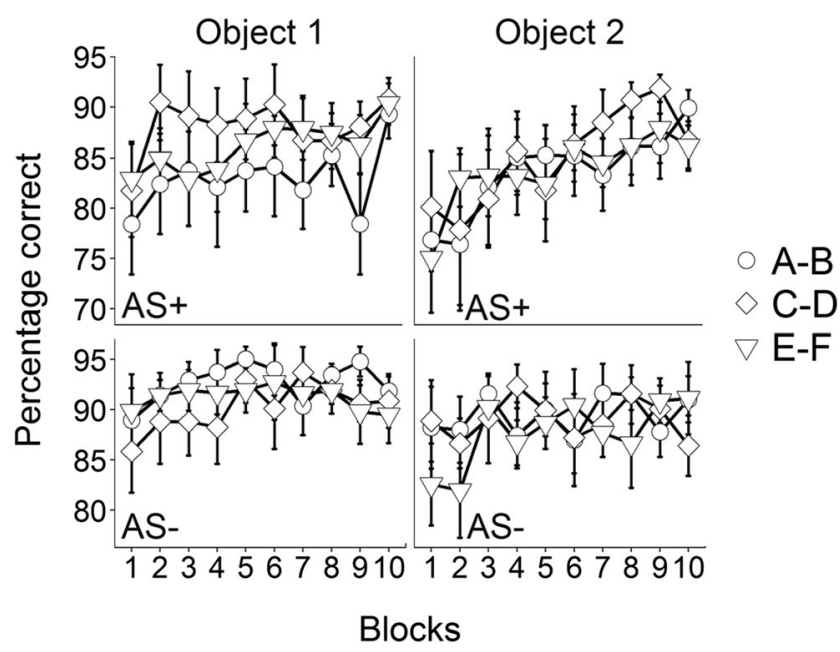

Fig. 5 Mean accuracy obtained during the training phase in baboons, depending on the group of baboons (AS+ and $\mathrm{AS}-$ ), 196-trial test block, and stimulus pair. The $y$-axes have different scales in the two groups and display only a subset of the range. Error bars represent the standard errors 
For the AS- group, the two-way ANOVA using the accuracy of the response to Object 1 revealed no significant main effect or interaction (all $p \mathrm{~s}>.05$ ), and these results were replicated in the analyses of the response to Object 2 (all $p \mathrm{~s}>$ $.05)$. Taken together, these data reinforce the idea that the baboons from $\mathrm{AS}^{-}$- did not learn that the stimuli were organized in pairs and were therefore unable to predict the identity of Object 2 depending on the identity of Object 1.

Response times of the test trials Figure 6 represents the mean response times obtained during the test in $\mathrm{AS}+$ and ASgroups, for each block and stimulus pair. With our procedure, we first expected that the effects of symmetry (if any) will be of maximal amplitude during the first test trials. These effects might eventually decrease in amplitude or even disappear because of the learning of the novel stimulus pairing that may occur with the repetition of the test blocks.

Analyses for the AS+ group To analyze the response times obtained for AS+ at the onset of the test, we computed twotailed paired $t$ tests comparing the mean RT2 obtained in the first 10 trials for the different stimulus pairs. These analyses indicated shorter RT2 for the stimulus pair E-D (mean $=674$ $\mathrm{ms}, S D=54 \mathrm{~ms}$ ) than for F-C (mean $=768 \mathrm{~ms}, S D=77 \mathrm{~ms}$ ), $t(7)=-4.17, p<.01$, Cohen's $d=0.58$. The differences between B-A (mean $=727 \mathrm{~ms}, S D=136 \mathrm{~ms}$ ) and E-D, or between B-A and F-C were not statistically significant ( $p s>.10)$. These results suggest that the AS+ baboons processed stimulus positional information. However, they provide no indication that these baboons were able to process the relation of symmetry.

To analyze how RT2 evolved with the repetition of the test blocks, we ran a block (1-5) by stimulus pair (B-A, F-C, E-D) two-way ANOVA on RT2 using blocks of 192-trials made from the grouping of two initial 96-trial blocks. This analysis showed a reliable effect of block, indicating that the RT2 decreased through continuous training, $F(4,28)=3.73, p<.02$, $\eta_{\mathrm{p}}{ }^{2}=.35$, but no effect of stimulus pair, $F(2,14)=2.60, p>$ .10 , and no reliable interaction $F(8,56)=1.24, p>.10$. Note the lack of effect of stimulus pair departs from the data obtained from the analysis of the 10 first trials. This discrepancy suggests that the effect of the preservation of the position of the stimuli in the training pairs declined with repeated testing.

Analyses for the AS- group The same analyses conducted on the AS- group revealed no difference in mean RT2 between the stimulus pairs when the first 10 test trials were considered (B-A: mean $=656 \mathrm{~ms}, S D=81 \mathrm{~ms} ; \mathrm{F}-\mathrm{C}$ : mean $=$ $664 \mathrm{~ms}, S D=38 \mathrm{~ms}$; E-D $=668 \mathrm{~ms}, S D=76 \mathrm{~ms}$; all $p \mathrm{~s}>.10$ ). In addition, the two-way ANOVA showed no reliable main effect of stimulus pair or block, and interaction between these two factors $(p s>.10)$.

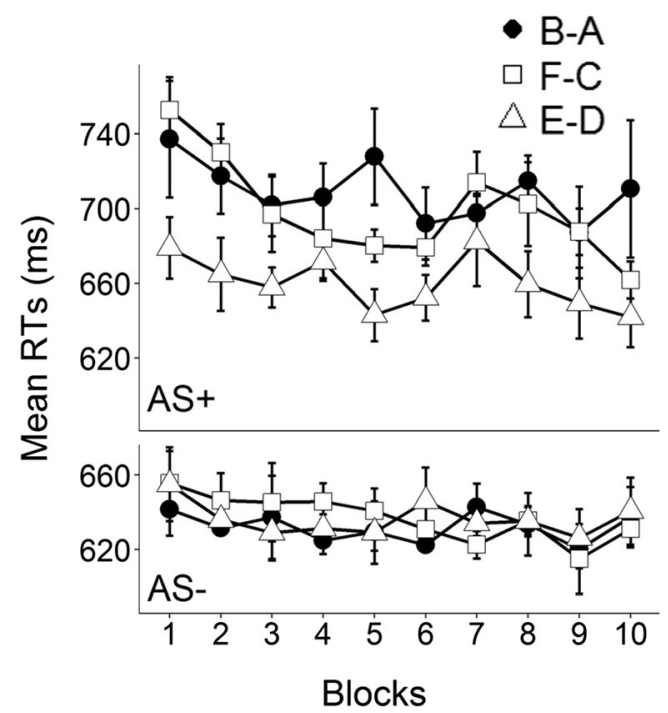

Fig. 6 Mean responses times on the second object (RT2) obtained in the $\mathrm{AS}+$ and AS - groups, for each stimulus pair and test block. The $y$-axes differ between the two groups. Error bars represent the standard errors centered on each participant mean RT

Accuracy of the test trials Figure 7 represents how the accuracy varied across the test blocks and stimulus pairs. Accuracy analyses followed the same logic as for the analyses of RT2. First, paired two-tailed $t$ tests compared the mean performance on Object 2 obtained during the first 10 test trials of each stimulus pair. The next analyses focused our attention on all the test blocks.

Analyses for the AS+ group This analysis revealed a trend for a greater performance at the onset of the test phase with $\mathrm{E}$ $\mathrm{D}($ mean $=92 \%$ correct, $\mathrm{SD}=11 \%$ ) than with both F-C (mean $=71 \%, S D=19 \%), t(7)=2.35, p=.05$, Cohen's $d=-0.37$, and B-A (mean $=66 \%, S D=30 \%), t(7)=2.20, p=.064$, Cohen's $d=-1.16$. No difference was found between F-C and

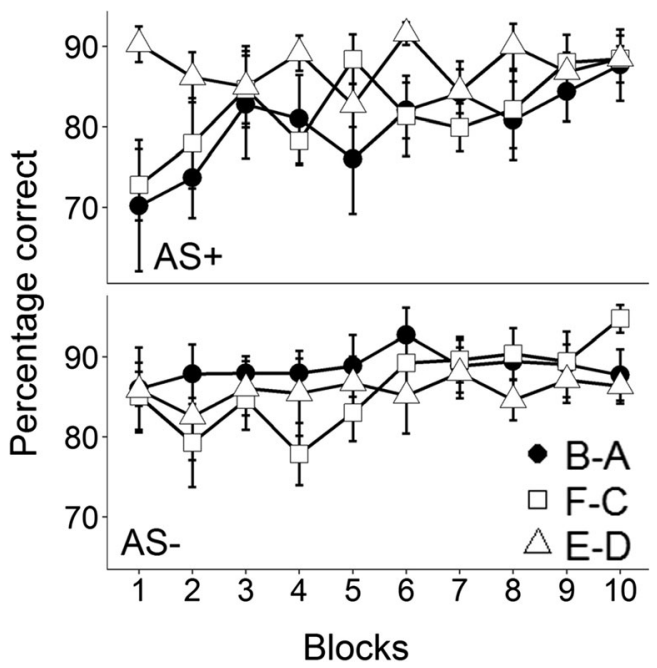

Fig. 7 Mean accuracy obtained in the two groups of baboons for each stimulus pair and test block. The $y$-axes differ between the two groups. Error bars represent the standard errors 
B-A $(p>.10)$. The results obtained from the analyses of the accuracy and mean RT2 were therefore highly consistent as the advantage found above for E-D in terms of response times converge with an accuracy advantage. Because the E-D pair is the sole test pair for which the order of the stimulus was preserved in comparison to training, this advantage for E-D confirms a processing of the positional information by the baboons from the AS+ group.

The second set of analyses on the five test blocks revealed a main effect of block, $F(4,28)=6.71, p<.001, \eta_{\mathrm{p}}{ }^{2}=.49$, as well as a significant block by pair interaction, $F(8,56)=2.34$, $p<.05, \eta_{\mathrm{p}}{ }^{2}=.25$. No main effect of stimulus pair was found $(p>.10)$. Post hoc analyses revealed that the baboons' performance with the E-D pair was higher compared to the B-A pair in the first test block, but that no difference was observed for the next blocks. These results therefore converge with those reported above on RT2. Altogether, these results indicate that the initial advantage for E-D in terms of RT and accuracy progressively disappeared during the test.

Analyses for the AS- group The analyses conducted on the data from the AS- group confirmed that no difference in accuracy was observed for this group during the first 10 test trials between the B-A (mean $=93 \%$ correct, $S D=6 \%$ ), F-C (mean $=91 \%$ correct, $S D=9 \%$ ), and E-D (mean $=87 \%$ correct, $S D=15 \%)$ pairs $(p \mathrm{~s}>.10)$. The two-way ANOVA considering the five blocks of tests showed no reliable main effect nor interaction $(p s>.10)$.

\section{Discussion}

This comparative human/baboon experiment examined the encoding of pairs of stimuli in sequences to identify potential species differences in their processing of the symmetry relation. A serial response time task involving pairs of objects was developed and presented to the two species for that purpose. In our task, we presumed that the individuals of the two species who learned the association between the objects to be touched on the displays should demonstrate an RT2 acceleration with training. We distinguished two groups of subjects on that basis and categorized all the individuals showing an RT2 acceleration during training in the AS+ group; all the remaining subjects were categorized in the AS- group.

The results of the test phase confirmed our expectation that the baboon and human subjects of the AS- groups did not process the associations between the target stimuli. In the AS- baboons, we obtained no reliable effect of stimulus pair and no Block $\times$ Stimulus Pair interaction in the test phase. We propose that the baboons of the AS- group only processed the blink in each display but did not process the fact that the stimuli which blinked once within a trial were organized in pairs. In the AS- humans, the only effect emerging from the test trials is a response time advantage for the E-D pair occurring at the onset of the test. This advantage suggests that the AS- human subjects encoded at least minimally the identity of the stimuli and their position. The processing of the positional information might therefore occur in the AS- human group along with the processing of the information on the blink mentioned above for the baboons.

Test trials identified the cognitive strategy employed by the members of the AS+ groups of each species. In humans, we found shorter RT2 in the B-A than in the F-C pair. The shorter RT2 obtained for B-A in Test Block 1, in comparison to F-C, can be explained by the preservation of the associative link between the stimuli in the B-A pair only. We take this result as demonstrating that humans from the AS+ group processed the associations between the stimuli, and furthermore processed A-B and B-A as symmetrical relations in our study. Importantly, humans from this AS+ group also demonstrated a RT2 advantage for E-D over B-A. This advantage for E-D suggests that this group also processed positional information, along with the information of the associations.

These results in humans contrast sharply with those obtained in baboons from the AS+ group. In these baboons, the stimulus pair giving rise to the shortest RT2 was E-D, not B$\mathrm{A}$, as found in humans. We also found a reliable difference between E-D and F-C. The advantage for the stimulus pair E$\mathrm{D}$, which emerged in the first 10 trials, is confirmed by a better accuracy for E-D, in comparison to both F-C and B-A. Critically, neither E-D nor F-C preserved the relations learned during the training phase, and the positional information is preserved in E-D only. Therefore, while baboons from the AS+ group processed the associations between the stimuli in the training phase, they did not processed A-B and B-A as symmetrical relations in our study. Therefore, our results suggest an encoding of positional information in baboons of the AS+ group. This encoding of the positional information is further confirmed by a lack of statistical difference between $\mathrm{B}-\mathrm{A}$ and $\mathrm{F}-\mathrm{C}$, which are the two stimulus pairs reversing their position in comparison to training. In turn, results suggest that the baboons from the AS- group have neither encoded the positional nor the relational information. Apparently, these subjects only paid attention to the blink cue, as discussed above.

The fact that the baboons from the AS+ group give priority to the positional information converges with previous findings and conclusions from Urcuioli (2015). Our results also add to the literature this idea that prioritization of positional over relational information is a pervasive effect that can be replicated in pigeons and monkeys, at least within the nonhuman animal species. The demonstration that nonhuman animals give priority to perceptual cues over relational ones is not new in the comparative literature. Such effects were already found with relational stimuli of different types (hierarchical stimuli: Fagot \& Deruelle, 1997; artificial relational stimuli: 
Fagot, Kruschke, Dépy, \& Vauclair, 1998; Wasserman, Fagot, \& Young, 2001; ABA/ABB sequential patterns: Chen, van Rossum, \& ten Kate, 2015). Nevertheless, our study makes it even more striking, following Urcuioli (2015), that such prioritization has major impacts on the nonhuman animals' ability to both processes symmetry and to form equivalence classes based on symmetrical relations.

The current research has practical consequences for further studies on symmetry and equivalence class formation in animals. As already suggested by Urcuioli (2015), it confirms that the search for symmetry in animals requires a strict control of the positional cues that might be available in the task. One limitation of our study is that spatial and temporal/ordinal information covaried during the training and test phases, because the first stimulus of each pair was always presented on the left side of the screen, the second one being presented on the right side. Our study therefore provides no information to distinguish the exact influence of these two factors (but see Lionello-DeNolf, 2009, for a discussion of this issue). Nevertheless, the current results as well as those of previous research all point to the same conclusion: They suggest that this kind of perceptual cues are very important to consider in future experimental designs. Note, however, that such effects do not rule out the possibility that other factors also intervene. For instance, Medam et al. (2016) proposed that experimental designs mixing symbolic and identity matching trials (as in Frank \& Wasserman, 2005) promote cognitive flexibility, because the subjects can learn that the stimuli can have different roles in the task. We suggest that a more flexible encoding of the stimulus pair should also be favored in future experimental designs.

The cognitive ability to solve symmetry problems seems highly adaptive, and experts of primate cognition can easily mention situations in which animals apparently treat relations between items as symmetrical. One classic example of this kind is the case of vervet monkeys, who can both produce alarm calls in response to leopards (leopard $\rightarrow$ alarm call) and can meanwhile show specific behavioral responses when hearing these calls, suggesting that they also treat the call $\rightarrow$ leopard symmetrical association (Seyfarth, Cheney, \& Marler, 1980). Although this kind of situations probably occurs in nature, they are neither as variable nor as frequent as found during interpersonal verbal communication in humans involving a continuous shift between speech production and speech perception. When communicating verbally with others, our comprehension of others' speech requires that the words we perceived are mapped to their referent, while speech production requires the reversed referent to word mapping. In other words, verbal communication is an exercise for which it is central to treat the associations/relationships between and among items as being bidirectional. This importance of symmetry for our communicative system might explain previous demonstrations of a strong relationship between the ability to develop equivalence classes and language skills (e.g., Devany et al., 1986). An important result in our study is that the AS+ humans who used labels to code the stimuli demonstrated symmetry. By contrast, the AS+ baboons and AS- humans who did not use labels to code the stimuli failed to process the stimulus pairing and relied exclusively on positional cues to solve the task. These results suggest an effect of stimulus labeling on the processing of the task. More experimental work will be needed to better understand this effect.

Most of the associations occurring in the environment are unidirectional. This is true in both humans and nonhuman animals (e.g., a horn predicts a car in humans, the animal keeper predicts food delivery in baboons, and the reverse associations are not true), but only humans have extra training with symmetrical relations via language. Other animal species that are missing a human-like communication system, and thus the extensive bidirectional training that humans have with their exposure to language, may require specific training and test conditions to learn that the stimuli go together in a bidirectional way; to neglect information such as the stimuli position, which is irrelevant to the task; and to be able to derive untrained (symmetrical) relations from the trained ones.

Acknowledgements The authors thank the staff of the CNRS primate center (Rousset-sur-Arc, France) as well as Julie Gullstrand for technical supports. This research was supported by the project Premilang2 (\#ANR13-BSH2-0002), labex BLRI (ANR-11-LABX-0036), and the Convergence ILCB institute.

\section{Compliance with ethical standards}

Ethical standard This research conformed to the Standard of the American Psychological Association's Ethical Principles of Psychologist and Code of Conduct, and received ethical approval from the French Ministry of Education (approval APAFIS\#2717$2015111708173794 \mathrm{v} 3$ ).

\section{References}

Adams, B. J., Fields, L., \& Verhave, T. (1993). Formation of generalized equivalence classes. Psychological Record, 43, 553-566.

Barros, R. S., Galvão, O. F., \& McIlvane, W. J. (2002). Generalized identity matching-to-sample in Cebus apella. The Psychological Record, 52, 441-460.

Boelens, H., van den Broek, M., \& van Klarenbosch, T. (2000). Symmetric matching to sample in 2-year-old children. Psychological Record, 50, 293-304.

Carp, C. L., \& Petursdottir, A. L. (2015). Intraverbal naming and equivalence class formation in children. Journal of the Experimental Analysis of Behavior, 104, 223-240.

Carr, D., Wilkinson, K. M., Blackman, D., \& Mcllvane, W. J. (2000). Equivalence classes in individuals with minimal verbal repertoires. Journal of Experimental Analysis of Behavior, 74, 101-114.

Chen, J., van Rossum, D., \& ten Cate, C. (2015). Artificial grammar learning in zebra finches and human adults: XYX versus XXY. Animal Cognition, 18(1), 151-164. doi:10.1007/ s10071-014-0786-4 
D’Amato, M. R., Salmon, D. P., Loukas, E., \& Tomie, A. (1985). Symmetry and transitivity of conditional relations in monkeys (Cebus apella) and pigeons (Columba livia). Journal of the Experimental Analysis of Behavior, 44, 35-47.

Devany, J. M., Hayes, S. C., \& Nelson, R. O. (1986). Equivalence class formation in language-able and language-disabled children. Journal of the Experimental Analysis of Behavior, 46, 243-257.

Eikeseth, S., \& Smith, T. (1992). The development of functional and equivalence classes in high-functioning autistic children: The role of naming. Journal of Experimental Analysis of Behavior, 58, 123-133.

Fagot, J., \& Bonté, E. (2010). Automated testing of cognitive performance in monkeys: Use of a battery of computerized test systems by a troop of semi-free ranging baboons. Behavior Research Methods, 42, 507-516.

Fagot, J., \& Deruelle, C. (1997). Processing of global and local visual information and hemispheric specialization in humans (Homo sapiens) and baboons (Papio papio). Journal of Experimental Psychology: Human Perception and Performance, 23, 429-442.

Fagot, J., Kruschke, J. K., Dépy, D., \& Vauclair, J. (1998). Associative learning in humans (Homo sapiens) and baboons (Papio papio): Species differences in learned attention to features. Animal Cognition, 1, 123-133.

Fagot, J., \& Paleressompoulle, D. (2009). Automatic testing of cognitive performance in baboons maintained in social groups. Behavior Research Methods, 41, 396-404.

Frank, A. J., \& Wasserman, E. A. (2005). Associative symmetry in the pigeon after successive matching-to-sample training. Journal of the Experimental Analysis of Behavior, 84, 147-165.

Kastak, C. R., Schusterman, R. J., \& Kastak, D. (2001). Equivalence classification by California sea lions using class-specific reinforcers. Journal of the Experimental Analysis of Behavior, 76, 131-158.

Kuno, H., Kitadate, T., \& Iwamoto, T. (1994). Formation of transitivity in conditional matching to sample by pigeons. Journal of the Experimental Analysis of Behavior, 62, 399-408.

Lionello-DeNolf, K. M. (2009). The search for symmetry: 25 years in review. Learning \& Behavior, 37, 188-203.

Lipkens, G., Hayes, S. C., \& Hayes, L. J. (1993). Longitudinal study of derived stimulus relations in an infant. Journal of Experimental Child Psychology, 56, 201-239.

Lipkens, R., Kop, P. F., \& Matthijs, W. (1988). A test of symmetry and transitivity in the conditional discrimination performances of pigeons. Journal of the Experimental Analysis of Behavior, 49, 395-409.
Medam, T., Marzouki, Y., Montant, M., \& Fagot, J. (2016). Categorization does not promote symmetry in Guinea baboons (Papio papio). Animal Cognition, 19, 987-998.

Misyak, J. B., Christiansen, M. H., \& Bruce Tomblin, J. (2010). Sequential expectations: The role of prediction-based learning in language. Topics in Cognitive Science, 2(1), 138-153.

Nissen, M. J., \& Bullemer, P. (1987). Attentional requirements of learning: Evidence from performance measures. Cognitive Psychology, 19(1), 1-32.

O'Donnell, J., \& Saunders, K. J. (2003). Equivalence relations in individuals with language limitations and mental retardation. Journal of Experimental Analysis of Behaviour, 80, 131-157.

Pepperberg, I. M., \& Gordon, J. D. (2005). Number comprehension by a grey parrot (Psittacus erithacus), including a zero-like concept. Journal of Comparative Psychology, 119, 197-209.

Schusterman, R. J., \& Kastak, D. (1993). A California sea lion (Zalophus californianus) is capable of forming equivalence relations. Psychological Record, 43, 823-839.

Seyfarth, R. M., Cheney, D. L., \& Marler, P. (1980). Monkey responses to three different alarm calls: Evidence of predator classification and semantic communication. Science, 210, 801-803.

Sidman, M. (1971). Reading and auditory-visual equivalences. Journal of Speech and Hearing Research, 14, 5-13.

Sidman, M., Rauzin, R., Lazar, R., Cunningham, S., Tailby, W., \& Carrigan, P. (1982). A search for symmetry in the conditional discriminations of rhesus monkeys, baboons, and children. Journal of the Experimental Analysis of Behavior, 37, 23-44.

Sidman, M., \& Tailby, W. (1982). Conditional discrimination vs. matching to sample: An expansion of the testing paradigm. Journal of the Experimental Analysis of Behavior, 37, 5-22.

Sweeney, M. M., \& Urcuioli, P. J. (2010). Reflexivity in pigeons. Journal of the Experimental Analysis of Behavior, 94, 267-282.

Urcuioli, P. J. (2008). Associative symmetry, antisymmetry, and a theory of pigeons' equivalence-class formation. Journal of the Experimental Analysis of Behavior, 90, 257-282.

Urcuioli, P. J. (2015). A successful search for symmetry. Conductal, 3, 4-25.

Wasserman, E. A., Fagot, J., \& Young, M. E. (2001). Same-different conceptualization by baboons (Papio papio): The role of entropy. Journal of Comparative Psychology, 115, 42-52.

Zentall, T. R., Clement, T. S., \& Weaver, J. E. (2003). Symmetry training in pigeons can produce functional equivalences. Psychonomic Bulletin \& Review, 10, 387-391. 